УДК 621.311

\title{
РАСЧЕТ ПАРАМЕТРОВ УСТРОЙСТВА КОМПЕНСАЦИИ РЕАКТИВНОЙ МОЩНОСТИ СЕТИ ЭЛЕКТРОСНАБЖЕНИЯ С ТРАНСФОРМАТОРОМ НА ВИЗУАЛЬНОЙ МОДЕЛИ
}

Канд. техн. наук. Е. В. Ягуп

\section{РОЗРАХУНОК ПАРАМЕТРІВ ПРИСТРОЮ КОМПЕНСАЦІЇ РЕАКТИВНОӤ ПОТУЖНОСТІ МЕРЕЖІ ЕЛЕКТРОПОСТАЧАННЯ 3 ТРАНСФОРМАТОРОМ НА ВІЗУАЛЬНІЙ МОДЕЛІ}

Канд. техн. наук. К. В. Ягуп

\section{CALCULATION OF PARAMETERS OF REACTIVE POWER COMPENSATION DEVICE OF POWER SUPPLY LINE WITH TRANSFORMER ON THE VISUAL MODEL}

\section{PhD in Technical Science K. Iagup}

В статье исследованы процессы компенсаџии реактивной мощности в системе электроснабжения на визуальной модели, созданной в программе MATLAB. Компенсирующее устройство состочт из симметро-компенсирующих конденсаторов, подключенных клиниям электропередачи через понижающий трансформатор. Предварительно проведен расчет параметров трансформатора, которые и были заложены в модель. В результате компьютерных экспериментов было установлено, что при снижении коэффициента трансформачии величины емкостей конденсаторов увеличиваются. При этом достигается практически полная компенсаџия реактивной мощности и полное симметрирование токов 8 сети электроснабжения.

Ключевые слова: реактивная мощность, симметро-компенсирующие конденсаторы, коэффициент трансформации.

У статті досліджено процеси компенсації реактивної потужності в системі електропостачання на візуальної моделі, шзо створена в програмі MATLAB. Компенсуючий пристрій складається з симетро-компенсуючих конденсаторів, щзо були підключені до ліній електропередачі через понижуючий трансформатор. Попередньо проведено розрахунок параметрів трансформатора, які і були закладені в модель. В результаті комп'ютерних 
дослідів було встановлено, щзо при зниженні коефіцієнта трансформації величини ємностей конденсаторів збільшуються. При изьому досягається практично повна компенсація реактивної потужності і повне симетрування струмів в мережі електропостачання.

Ключові слова: реактивна потужність, симетро-компенсуючі конденсатори, коефіцієнт трансформації.

The article explores the possibility of reactive power compensation in the power supply system on the visual model created in the software package MATLAB. In the power supply system reactive power arises because of the asymmetric distribution of active-inductive load on phases of electrical network. In this case, a fairly large amplitude currents power lines are observed.

The compensating device is represented by symmetry-compensating capacitors connected to a power line through a step-down transformer. Previously calculations of transformer parameters required for its modeling in MATLAB were carried out: power, input and output voltages, winding resistances and leakage inductance, active and inductive resistances of magnetization branch.

The search for optimal parameters of symmetry-compensating device was carried out with using the built-in MATLAB function of search optimization fminsearch, which operates on NelderMead optimization algorithm. Computer experiments were performed for models with different ratios of transformation of symmetry-compensating device. As a result, it was found that the values of the capacitors increases with decreasing ratio of transformation. Almost complete compensation of reactive power, a significant reduction in the amplitude values of current networks and their full symmetry were achieved at the end of the optimization

Keywords: reactive power, compensation capacitors, unbalanced load, transformation ratio.

\section{Актуальность}

Внедрение в эксплуатацию таких современных устройств, как частотные преобразователи электроприводов, импульсные блоки питания, систем кондиционирования и освещения, а так же активное использование асинхронных двигателей и других электрических машин, приводит к возникновению реактивной мощности в трехфазных сетях. Передача в сеть реактивной мощности приводит к таким негативным последствиям, как неполное использование трансформаторов, первичных двигателей и генераторов электростанций, дополнительным активным потерям мощности и энергии в сети, увеличению потерь напряжения в линии электропередачи, перегреву проводов, что в свою очередь увеличивает удельный расход топлива [1]. Поэтому компенсация реактивной мощности в системах электроснабжения становится все более актуальной задачей.

Одним из наиболее экономичных и эффективных способов компенсации реактивной мощности является применение конденсаторных установок. Преимущества их использования состоят в небольших эксплуатационных затратах и простоте монтажа и обслуживания. В [2] была рассмотрена возможность полной компенсации реактивной мощности в системе с несимметричной активноиндуктивной нагрузкой на основе использования компенсирующего устройства, состоящего из межфазных конденсаторов. Однако в [2] не рассматривался случай, когда напряжение в линиях электропередачи может существенно превышать напряжение, подаваемое на компенсирующее устройство. В таком варианте целесообразно обеспечить питание симметро-компенсирующих конденсаторов через понижающий трансформатор.

\section{Цели и задачи исследования}

Целью статьи является расчет параметров симметро-компенсирующего устройства, подключаемого к сети через трансформатор, при помощи поисковой оптимизации на основе компьютерной модели трехфазной системы электроснабжения с компенсирующим устройством, состоящим из трансформатора и конденсаторов. 
Основная часть исследования

Рассмотрим трехфазную систему электроснабжения, к которой подключен трансформатор

$\mathrm{c}$

симметро-

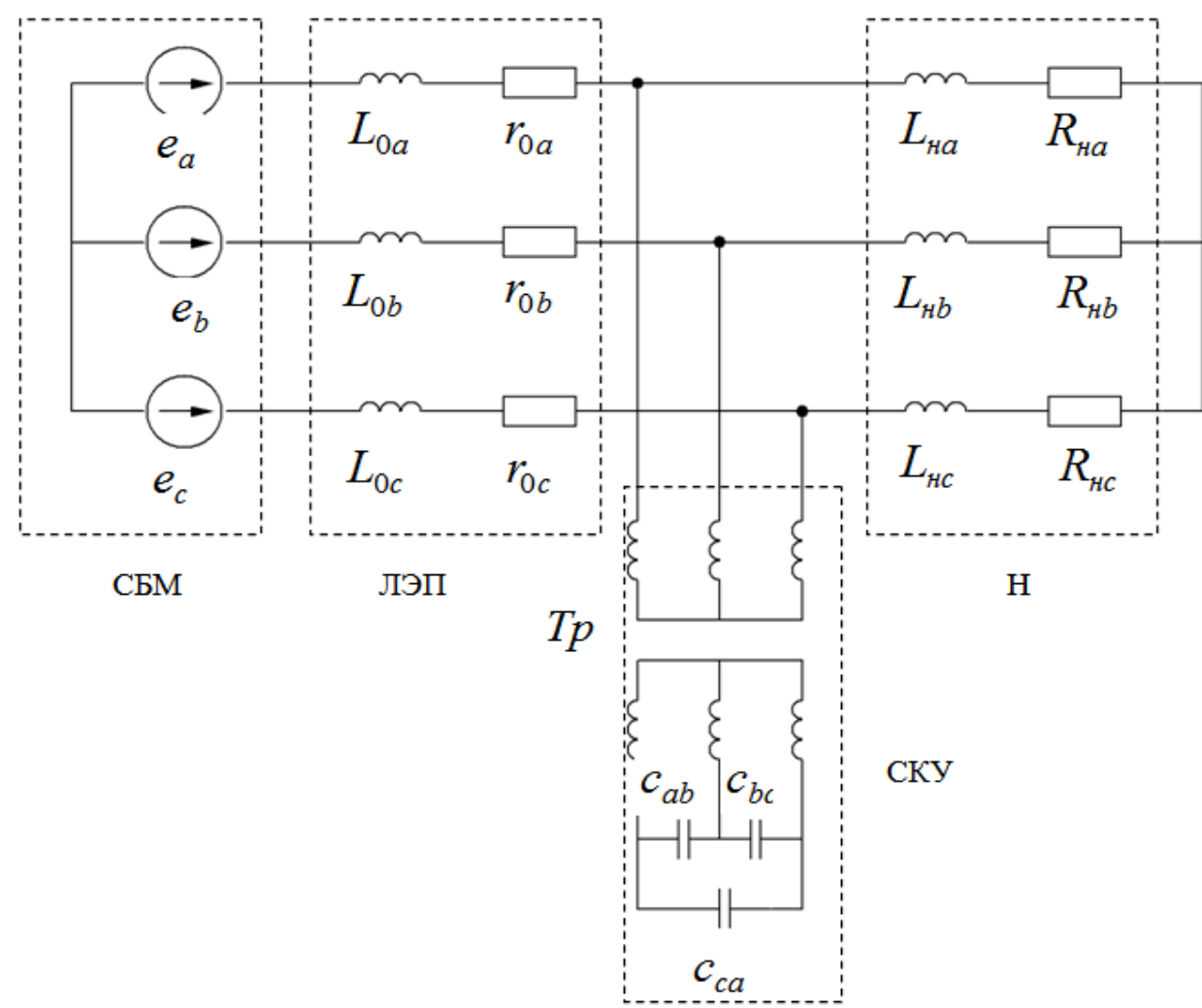

Рис. 1 - Трехфазная электрическая сеть с симметро-компенсирующим конденсаторным устройством компенсации реактивной мощности.

Амплитуды фазных напряжений источников сети бесконечной мощности (СБМ) равны $U_{a}=U_{b}=U_{c}=100 \mathrm{~B}$. Сопротивления и индуктивности в линии электропередачи (ЛЭП) соответственно приняты $r_{0 a}=r_{0 b}=r_{0 c}=0,1 O M$ $L_{0 a}=L_{0 b}=L_{0 c}=0,001 \Gamma \mu$. Нагрузка $(\mathrm{H})$ по фазам распределена неравномерно и характеризуется следующими значениями сопротивлений и индуктивностей:

$$
R_{a}=0.7 O M ; L_{a}=0,005 \Gamma \mu ; R_{b}=1 O M ; L_{b}=
$$

Первичные и вторичные обмотки трансформатора соединены в звезду, а конденсаторы соединены треугольником. Для модели рассчитаем номинальные параметры трансформатора: мощность, активные и индуктивные сопротивления обмоток. Расчет будем осуществлять исходя из рассчитанных в [2] значений емкостей симметрирующих конденсаторов в схеме без подключения трансформатора. Расчет номинальных параметров трансформатора осуществляется исходя из максимального тока нагрузки трансформатора при рассмотрении схемы системы электроснабжения без трансформатора. В [2] в результате оптимизации были получены следующие величины симметрирующих конденсаторов:

$$
X_{C}=\frac{1}{\omega C}=\frac{1}{2 \cdot \pi \cdot 50 \cdot 5,6209} 10^{6}=5,664 O M
$$


Амплитудное значение тока этого конденсатора

$$
I_{m}=\frac{U_{s}}{x_{C}}=\frac{\sqrt{3} * 100}{5,664}=30,5807 \mathrm{~A}
$$

Мощность в одной фазе представлена произведением линейного тока на линейное межфазное напряжение

$$
P_{a}=\frac{I_{m} U_{m}}{\sqrt{2} \cdot \sqrt{2}}=\frac{30,5807 \cdot 100}{2}=2648,363
$$

Номинальное значение мощности трансформатора:

$$
2648,363 * 3=7945,088 \text { В }
$$

Примем номинальную мощность трансформации 4:1, током холостого хода, трансформатора равной типовой 10000 Вт. составляющим $2 \%$ и напряжением короткого

Осуществим расчет внутренних замыкания 7\%, активные потери в опыте сопротивлений и индуктивностей холостого хода приняты $\mathrm{P}_{\mathrm{x}}=70$, активные трансформатора подключенным линейно к потери в опыте короткого замыкания линии электропередачи с коэффициентом $\mathrm{Pk}=600$.

Рассчитаем действующее линейное напряжение первичной обмотки:

$$
U_{1 H}=\frac{\sqrt{3} \cdot U_{\phi}}{\sqrt{2}}=\frac{\sqrt{3 \cdot} \cdot 100}{\sqrt{2}} 122,45 B
$$

Действующее линейное напряжение вторичной обмотки рассчитаем исходя из заданного коэффициента трансформации:

$$
U_{2 H}=\frac{U_{1 H}}{k}=\frac{122,45}{4}=30,619 B
$$

Мощность, приходящаяся на одну фазу:

$$
S_{1}=\frac{S_{H}}{3}=\frac{10000}{3}=3333,33 \mathrm{Bm}
$$

Действующее значение напряжения на первичной обмотке трансформатора:

$$
U_{1 f}=\frac{U_{1 H}}{\sqrt{m}}=\frac{122,48}{\sqrt{3}}=70,71 B
$$

Номинальный ток первичной обмотки трансформатора находим как отношение номинальной мощности к номинальному фазному напряжению:

$$
I_{1 H}=\frac{S_{1}}{U_{1 f}}=\frac{3333,33}{70,71}=47,14 \mathrm{~A}
$$

Номинальный ток во вторичной обмотке:

$$
I_{2 H}=\frac{I_{1 H} \cdot U_{1 H}}{U_{2 H}}=\frac{47,14 \cdot 122,474}{30,619}=188,562 \mathrm{~A}
$$

Рассчитаем фазное напряжение короткого замыкания

$$
U_{K f}=\frac{e_{K} \cdot U_{1 f}}{100}=\frac{7 \cdot 70,71}{100}=4,95 \mathrm{~B}
$$


Полное сопротивление короткого замыкания находим как отношение напряжения короткого замыкания к номинальному току.

$$
Z_{\kappa}=\frac{U_{K f}}{I_{1 \text { ном }}}=\frac{4,95}{47,14}=0,105 O M
$$

Активную составляющую сопротивления короткого замыкания рассчитаем по следующей формуле:

$$
R_{\kappa}=\frac{P_{K}}{3 I_{1 \text { ном }}^{2}}=\frac{600}{3 \cdot 47,14^{2}}=0,09 O_{\mathcal{M}}
$$

Найдем реактивную составляющую короткого замыкания .

$$
X_{\kappa}=\sqrt{Z_{K}^{2}-R_{K}^{2}}=\sqrt{0,105^{2}-0,09^{2}}=0,054 O M
$$

Активные и индуктивные сопротивления первичной обмотки трансформатора будем считать половине активного и индуктивного сопротивления короткого замыкания.

$$
\begin{aligned}
& R_{1}=\frac{R_{K}}{2}=0,045 O M \\
& X_{1}=\frac{X_{K}}{2}=0,027 O M
\end{aligned}
$$

Активное сопротивление вторичной обмотки в Т-образной схеме замещения:

$$
R_{2}=\left(\frac{U_{2 H}}{U_{1 H}}\right)^{2} \cdot R_{1}=0,002813 O M
$$

Индуктивность рассеивания первичной обмотки в Т-образной схеме замещения

$$
L_{1 S}=\frac{X_{1}}{\omega}=0,00008608 \Gamma \mu
$$

Индуктивность рассеивания вторичной обмотки в Т-образной схеме

$$
L_{2 s}=\left(\frac{U_{2 H}}{U_{1 H}}\right)^{2} \cdot L_{1 s}=0,00000538 \Gamma H
$$

Ток холостого хода в первичной обмотке:

$$
I_{x}=\frac{i_{x} \cdot I_{1 H}}{100}=0,94281 \mathrm{~A}
$$

Полное сопротивление холостого хода:

$$
Z_{x}=\frac{U_{1 \Phi}}{I_{x}}=75 O_{\mathcal{M}}
$$

Активное сопротивление холостого хода

$$
R_{x}=\frac{P_{x}}{m \cdot I_{x}^{2}}=26,25 O M
$$

Вычислим индуктивное сопротивление холостого хода:

$$
X_{x}=\sqrt{Z_{x}^{2}-R_{x}^{2}}=70,256
$$

Рассчитаем активное сопротивление, отражающее потери в стали сердечника: 


$$
R_{\mu}=R_{x}-R_{1}=26,205 O M
$$

Индуктивное сопротивление последовательной намагничивающей ветви

$$
X_{\mu}=X_{x}-X_{1 S}=70,229 O \mu
$$

Вычислим параметры параллельной намагничивающей ветви:

$$
\begin{aligned}
& R_{m}=R_{\mu}+\frac{X_{\mu}^{2}}{R_{\mu}}=214,419 O M ; \\
& X_{m}=X_{\mu}+\frac{R_{\mu}^{2}}{X_{\mu}}=80,007 O M .
\end{aligned}
$$

Таким образом, вычислены все параметры трансформатора, которые далее будут подставлены в модель MATLAB.

Реализация компьютерной модели трехфазной системы электроснабжения с компенсирующей системой, которая позволяет определить оптимальные условия компенсации реактивной мощности. приведена на рис. 2. Параметры модели соответствуют указанным ранее и рассчитанным значениям.

В модели мгновенные значения токов и напряжений измеряются виртуальными амперметрами и вольтметрами и передаются специальным блокам расчета реактивных мощностей фаз $\mathrm{Sa}, \mathrm{Sb}$ и $\mathrm{Sc}$. Затем вычисляется среднеквадратичный функционал реактивных мощностей, для чего используются блоки умножителей и сумматора, который и принимается за целевую функцию. Значение целевой функции передается в рабочее пространство MATLAB, где используется встроенной файл-функцией оптимизации fminsearch(). В процессе оптимизации значение целевой функции сводится к минимуму посредством изменения значений емкостей конденсаторов $\mathrm{Cab}, \mathrm{Cbc}$ и Cca. При проведении экспериментов был использован метод stiff23s Розенброка, шаг интегрирования 0.0001 с, что позволило проводить расчеты с достаточно высокой точностью. Время прогона модели составляет 0.06 с, что является достаточным для выхода модели на установившийся

режим

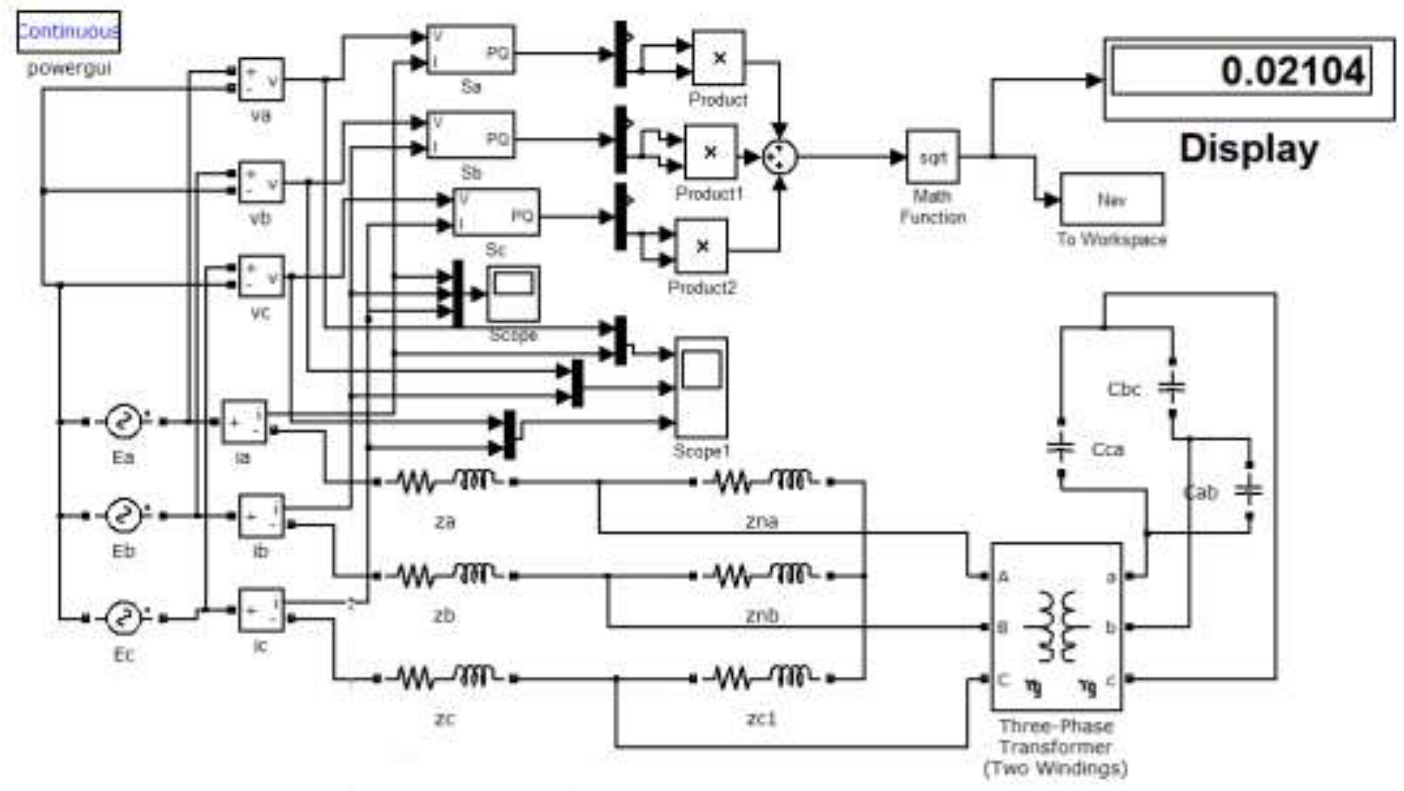

Рис. 2 - Визуальная модель трехфазной системы электроснабжения с симметрирующим устройством 


\section{Результаты исследования}

Начальные значения параметров оптимизации принимаются равными 10 мкФ, при этом начальное значение целевой функции составляет 1199. При этом наблюдался большой разброс в значениях амплитуд токов в фазах. Также следует отметить, что значения амплитуд токов были достаточно велики превышая 30 А (рис. 3).

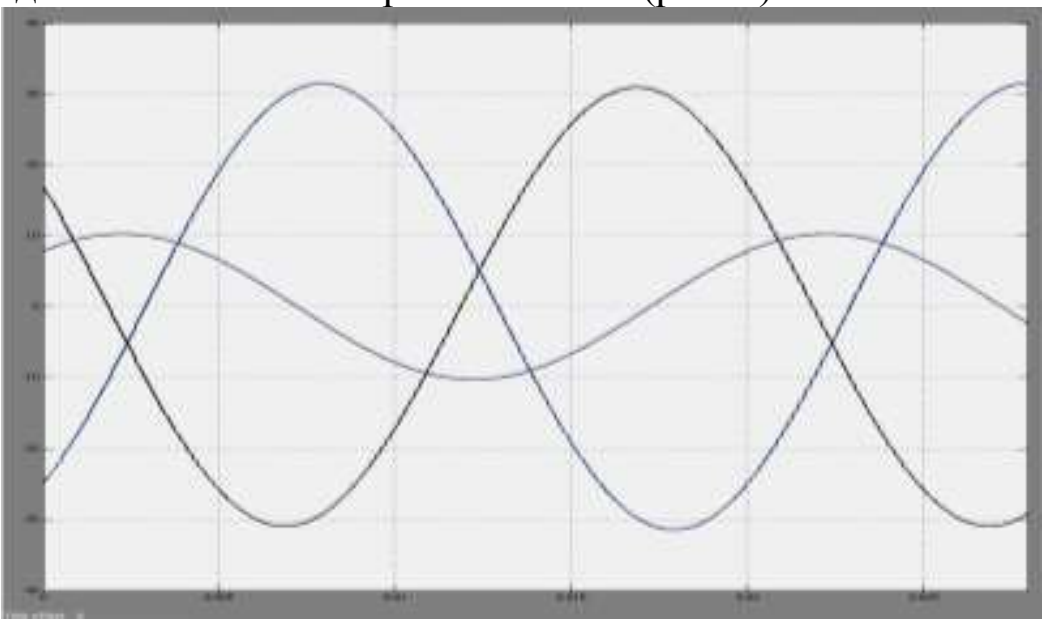

Рис. 3 - Временные диаграммы сетевых токов до оптимизации

Оптимизация проводилась для трансформации трансформатора. Результаты нескольких вариантов работы модели, в оптимизации сведены в таблицу 1. которых менялся коэффициент

Таблица 1. - Результаты моделирования

\begin{tabular}{|c|c|c|c|}
\hline $\begin{array}{c}\text { Коэффициент } \\
\text { трансформации }\end{array}$ & \multicolumn{1}{|c|}{$C_{a b}$} & $C_{b c}$ & $C_{c a}$ \\
\hline $100: 100$ & $5,5980 \mathrm{e}-004$ & $2,0741 \mathrm{e}-004$ & $4,0230 \mathrm{e}-005$ \\
\hline $100: 50$ & 0,0022 & $8,2962 \mathrm{e}-004$ & $1,6092 \mathrm{e}-004$ \\
\hline $100: 25$ & 0,0090 & 0,0033 & $6,4397 \mathrm{e}-004$ \\
\hline
\end{tabular}

В результате проведенных исследований снижение коэффициента трансформации приводило к возрастанию значений емкостей конденсаторов, что отображено на рис. 4

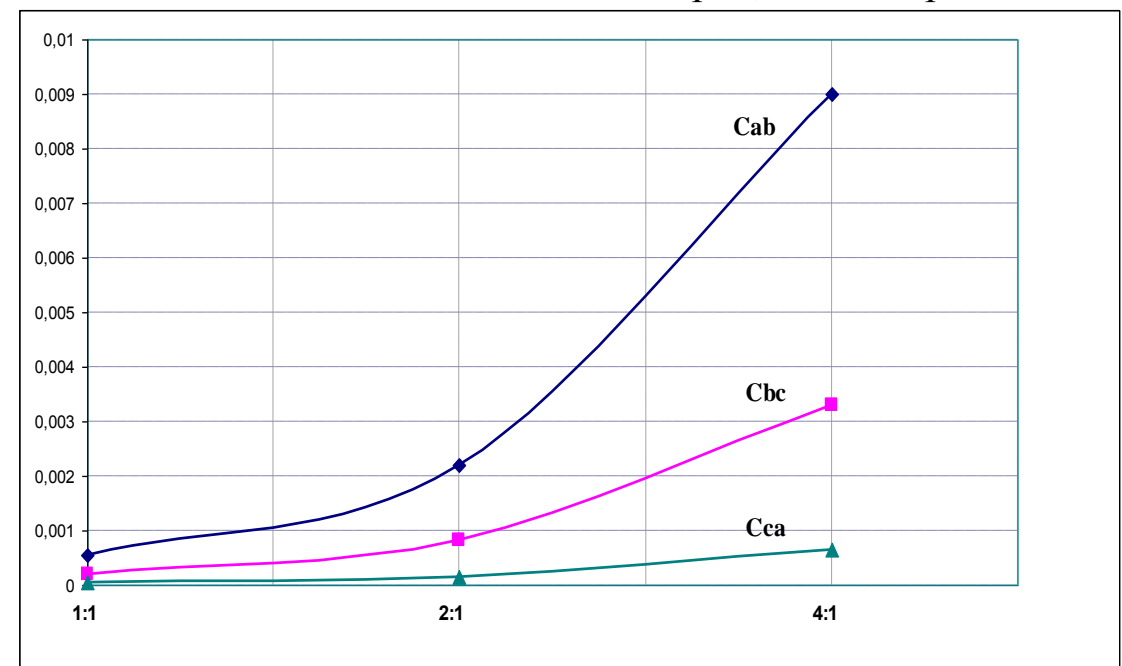

Рис. 4. - Зависимость значений емкостей конденсаторов от коэффициента трансформации 
На рис. 5 приведены графики фазных токов после установления режима компенсации. Из них видно, что их амплитуды стали равными по величине $(7,8$
А), а фазы в точности совпадают с фазами питающих напряжений, что свидетельствует о том, что источники отдают лишь активную мощность.

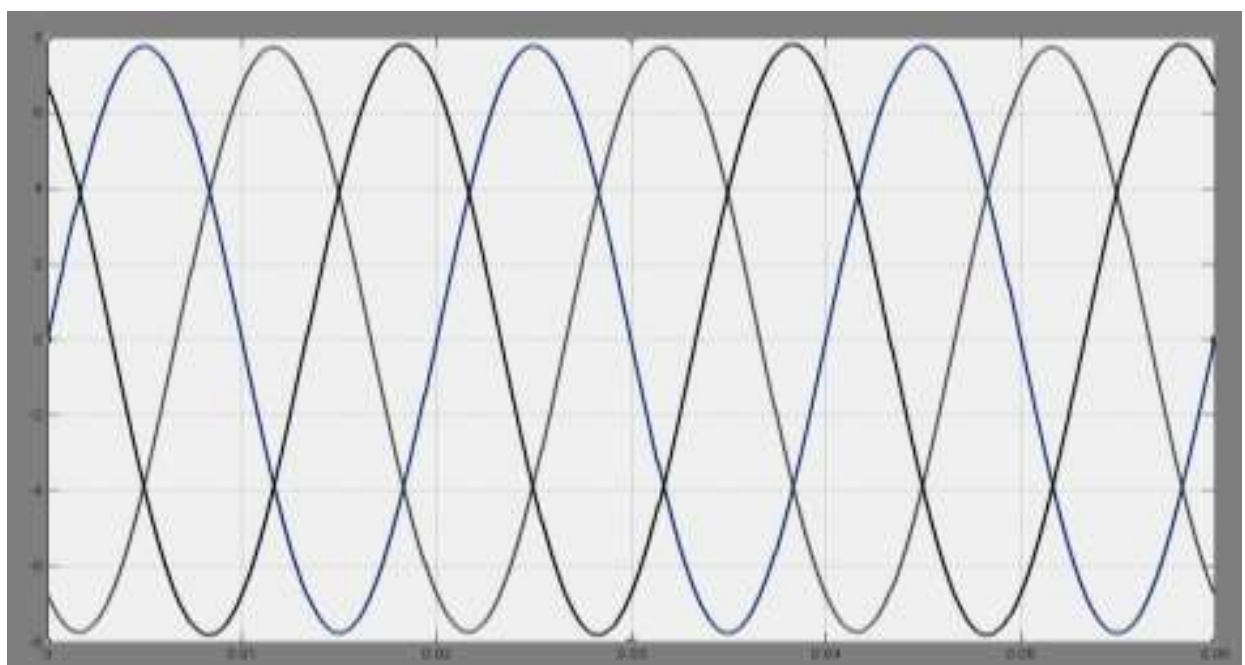

Рис. 5 - Временные диаграммы сетевых токов после оптимизации при коэффицинте трансформации 4:1.

\section{Выводы.}

1. Исследована возможность подключения симметро-компенсирующего

конденсаторного устройства к системе электроснабжения через понижающий трансформатор, что может быть применено в тех случаях, когда напряжение системы достаточно высоко.

2. Путем моделирования при помощи алгоритмов поисковой оптимизации достигнута компенсация реактивной мощности и полное симметрирование в исследуемой системе электроснабжения.
3. Рассчитываемые предложенным методом параметры симметро-компенсирующего устройства обеспечивают совершенные компенсацию и симметрирование с учетом реактивного и несимметричного характера нагрузки, а так же реактивностей линии электропередачи и трансформатора симметрокомпенсирующего устройства.

4. Результаты расчетов представлены в виде графиков, отражающих для принятой нагрузки изменения емкостей конденсаторов в зависимости от коэффициента трансформации понижающего трансформатора.

\section{Литература:}

1. Минин Г.П. Реактивная мощность. - М. Энергия, 1978. - 88 с.

2. Ягуп В.Г. Ягуп, В.Г. Расчет режима компенсации реактивной мощности в несимметричной системе электроснабжения методом поисковой оптимизации [Текст] // В. Г. Ягуп, Е. В. Ягуп // Наукові праці Донецького національного технічного університету. Серія «Електротехніка і енергетика». - 2011. - Вип. 11 (186). - С. 449-454.

3. Nelder, J. A. A Simplex Method for Function Minimization [Text] / J. A. Nelder, R. Mead // Computer J. - 1965. - Vol. 7, Issue 4. - P. 308-313

4. Дьяконов, В. П. MATLAB 6/6.1/6.5 + Simulink 4/5. Основы применения. Полное руководство пользователя [Текст] /В. П. Дьяконов. - М.: СОЛОН-Пресс, 2002. - 768 с. Рецензент д-р техн. наук, профессор Я.В.Щербак

Ягуп Катерина Валеріївна, канд. техн. наук, доцент кафедри автоматизованих систем електричної тяги Українського державного університету залізничного транспорту. Тел. (057)730-10-76

Iagup Katerina Valeriivna, cand. of techn. Sciences, the senior lecture of Department of automated electric transport systems of the Ukrainian State University of Railway Transport. Tel. (057)730-10-76 